\title{
Intrinsic noise improves speech recognition in a computational model of the auditory pathway
}

\author{
Achim Schilling ${ }^{1,2}$, Richard Gerum ${ }^{3}$, Alexandra Zankl ${ }^{1,2,4}$, Claus Metzner $^{1,3}$, Andreas Maier ${ }^{5}$, and \\ Patrick Krauss ${ }^{1,2,4,6}$ \\ ${ }^{1}$ Neuroscience Lab, University Hospital Erlangen, Germany \\ ${ }^{2}$ Cognitive Computational Neuroscience Group, Chair of English Philology and Linguistics, \\ Friedrich-Alexander University Erlangen-Nürnberg (FAU), Germany \\ ${ }^{3}$ Chair of Biophysics, Friedrich-Alexander University Erlangen-Nürnberg (FAU), Germany \\ ${ }^{4}$ Linguistics Lab, Friedrich-Alexander University Erlangen-Nürnberg (FAU), Germany \\ ${ }^{5}$ Chair of Machine Intelligence, Friedrich-Alexander University Erlangen-Nürnberg (FAU), Germany \\ ${ }^{6}$ University Medical Center Groningen, University of Groningen, The Netherlands
}

July 24, 2020

\section{Keywords:}

speech processing, hearing loss, auditory perception, stochastic resonance, deep neural networks, dorsal cochlear nucleus, perceptive phonetics, machine learning

\begin{abstract}
Noise is generally considered to harm information processing performance. However, in the context of stochastic resonance, noise has been shown to improve signal detection of weak subthreshold signals, and it has been proposed that the brain might actively exploit this phenomenon. Especially within the auditory system, recent studies suggest that intrinsic noise plays a key role in signal processing and might even correspond to increased spontaneous neuronal firing rates observed in early processing stages of the auditory brain stem and cortex after hearing loss. Here we present a computational model of the auditory pathway based on a deep neural network, trained on speech recognition. We simulate different levels of hearing loss and investigate the effect of intrinsic noise. Remarkably, speech recognition after hearing loss actually improves with additional intrinsic noise. This surprising result indicates that intrinsic noise might not only play a crucial role in human auditory processing, but might even be beneficial for contemporary machine learning approaches.
\end{abstract}




\section{Introduction}

The term noise usually describes undesirable disturbances or fluctuations, and is considered to be the "fundamental enemy" [1] for communication and error-free information transmission and processing in engineering. However, a vast and still increasing number of publications demonstrate the various benefits of noise for signal detection and processing, among which the most important phenomena are called stochastic resonance [1], coherence resonance [2], and recurrence resonance [3].

The term stochastic resonance (SR), first introduced by Benzi et al. [4], refers to a processing principle in which signals that would otherwise be sub-threshold for a given sensor can be detected by adding a random signal of appropriate intensity to the sensor input [4-6]. SR occurs ubiquitously in nature and covers a broad spectrum of systems in physical and biological contexts [1,7]. Especially in neuroscience, it has been demonstrated to play an essential role in a vast number of different systems [8-16]. Also, it has already been proposed that spontaneous random activity, i.e. noise, may increase information transmission via SR in the auditory brain stem [17].

In self-adaptive signal detection systems based on SR, the optimal noise intensity is continuously adjusted via a feedback loop so that the system response remains optimal in terms of information throughput, even if the characteristics and statistics of the input signal change. The term adaptive SR was coined for this processing principle [18-20]. In a previous study we demonstrated that the auto-correlation of the sensor output, a quantity always accessible and easy to analyze by neural networks, can be used to quantify and hence maximize information transmission even for unknown and variable input signals [21].

In further studies we demonstrated theoretically and empirically that adaptive SR based on output auto-correlations might be a major processing principle of the auditory system that serves to partially compensate for acute or chronic hearing loss, e.g. due to cochlear damage [22-25]. Here, the noise required for SR would correspond to increased spontaneous neuronal firing rates in early processing stages of the auditory brain stem and cortex, and would be perceived as a phantom perception. Remarkably, this phenomenon has frequently been observed in animal models and in humans with subjective tinnitus [26-29], which in turn is assumed to be virtually always caused by some kind of apparent [30-33] or hidden hearing loss [34,35]. From this point of view, phantom perceptions like tinnitus seem to be a side effect of an adaptive mechanism within the auditory system whose primary purpose is to compensate for reduced input through continuous optimization of information transmission [22,24,25].

The dorsal cochlear nucleus (DCN) was shown to be the earliest processing stage where acoustic trauma causes increased spontaneous firing rates [29,36-38]. Interestingly, this increase in spontaneous activity, i.e. neural hyperactivity, is correlated with the strength of the behavioral signs of tinnitus in animal models $[39,40]$. Furthermore, the hyperactivity is localized in those regions of the DCN that are innervated by the damaged parts of the cochlear [41]. Gao and colleagues [42] recently described changes in DCN fusiform cell spontaneous activity after noise exposure that supports the proposed SR mechanism. In particular, the time course of spontaneous rate changes shows an almost complete loss of spontaneous activity immediately after loud sound exposure (as no SR is needed due to stimulation that is well above threshold), followed by an overcompensation of spontaneous rates to levels well above pre-exposition rates since SR is now needed to compensate for acute hearing loss [42]. It is well known that the DCN receives not only auditory input from the cochlea, but also from the somatosensory system $[29,43,44]$, and that noise trauma alters long-term somatosensory-auditory processing in the DCN [45], i.e. somatosensory projections are up-regulated after deafness [46].

Therefore, we previously proposed the possibility that the neural noise for SR is injected into the auditory system via somatosensory projections to the DCN $[22,24,25]$. The idea that SR plays 
a key role in auditory processing and actually takes place in the DCN is supported by a number of findings. For instance, it is well known, that jaw movements lead to a modulation of subjective tinnitus loudness [47]. This may easily be explained within our framework, as jaw movements alter somatosensory input to the DCN. Since this somatosensory input corresponds to the noise required for SR, auditory input to the $\mathrm{DCN}$ is modulated through this mechanism, and the altered noise level is then perceived as modulated tinnitus [22, 24, 25]. Along the same line, one may explain why both, the temporo-mandibular joint syndrome and whiplash, frequently cause so called somatic tinnitus [48]. Another example is the finding of Tang et al. [49,50], who demonstrated that somatosensory input and hence tinnitus sensation may also be modified by serotonergic regulation of excitability of principal cells in the DCN. In addition, DCN responses to somatosensory stimulation are enhanced after noise-induced hearing loss [51,52]. Finally, and most remarkable, electro-tactile stimulation of finger tips, i.e. increased somatosensory input, significantly improves both, melody recognition [53] and speech recognition [54] in patients with cochlear implants.

In order to further support the hypothesis that SR plays a key role in auditory processing and takes place in the DCN, we here present a hybrid computational model of the auditory pathway, trained on speech recognition. In particular, we simulate different levels of hearing loss (cochlear damage) and compare the resulting accuracies for speech recognition with the accuracy of the nondisturbed model (i.e. without simulated hearing loss). As expected, we find that the accuracy decreases systematically with increasing hearing loss.

Subsequently, we add intrinsic noise of different intensities to the model. Here, we find SR-like behavior for all levels of hearing loss: depending on the intensity of the noise, accuracy first increases, reaches a peak, and finally decreases again. This means that speech recognition after hearing loss may indeed be improved by our proposed mechanism. This intriguing result indicates, that SR indeed plays a crucial role in auditory processing, and might even be beneficial for contemporary machine learning approaches. 


\section{Results}

\section{Layout of the computational model and general approach}

The model comprises three modules (Figure 1): (1) an artificial cochlea modeled as an array of band-pass filters, (2) a model of the dorsal cochlear nucleus (DCN), implemented as an array of leaky integrate-and-fire (LIF) neurons, and (3) a deep neural network, that represents all further processing stages beyond the DCN up to the auditory cortex and higher, language associated, cortex areas.

The input to the model are single words of spoken language encoded as wave files with a sampling rate of $44.1 \mathrm{kHz}$ and $1 \mathrm{~s}$ duration (Figure 1a, cf. Methods). These wave files represent the acoustic input of speech to the auditory system, and are processed in the first module of the model representing the cochlea and the spiral ganglion (Figure 1b). Like in previously published models [55-57], this module is implemented as an array of rectangular band-pass filters. In order to limit the total computation time, we restricted our model to 30 band-pass filters, instead of the actual amount of approximately 3500 inner hair cells in the human cochlea [58]. According to the physiology of the cochlea [59], the center frequencies of the band-pass filters are chosen such that they cover the frequency range from $100 \mathrm{~Hz}$ to $10 \mathrm{kHz}$ in logarithmic steps (cf. Methods).

The continuous multi-channel output of the band-pass filter array serves as input to an array of 30 LIF neurons [60] representing the DCN (Figure 1c). We here applied a one-to-one mapping from band-pass filters to model neurons, i.e. we do not explicitly account for putative cross-talk between neighboring frequency channels. However, since both the cochlea and the DCN model only consist of 30 different frequency channels, each of these channels may be regarded as an already coarse grained version of approximately 100 different frequency channels that exist in the human auditory system. Thus, eventual cross-talk is implicitly implemented in our model within each of the 30 modelled channels. The output of our DCN model comprises the spike trains of the 30 LIF neurons. Note that, in our DCN model, a single IF neuron represents approximately ten biological neurons processing the same frequency channel [61].

In our cochlea and DCN model, the outputs of the band-pass filters and the membrane potentials of the LIF neurons change with the same rate $(44.1 \mathrm{kHz})$ as the wave file input. However, the LIF neurons spike at lower average rates, due to their refractory period. It is therefore possible to downsample this sparse output spike train, thereby reducing the data volume for the subsequent deep neural network. In order to preserve enough temporal information for phase coding, we down-sample the DCN output only by a factor of five, so that the 44100 momentary amplitudes of the input wave file per second are finally transformed into a binary $30 \times 8820$ matrix.

These binary matrices serve as training input for the deep neural network, representing all further processing stages beyond the DCN up to the auditory cortex and higher, language associated, cortex areas. The neural network consists of four convolutional layers and three fully connected layers, and is trained with error backpropagation on the classification of 207 different German words (Figure 1d). The resulting classification accuracy of the trained network serves as a proxy for speech recognition (Figure 1e).

In order to simulate a particular hearing loss, the output amplitudes of the cochlea model are decreased by a certain factor, independently for the different frequency channels (Figure 1f). Subsequently, these modified cochlea outputs are further processed in the LIF neurons, finally resulting in a new binary matrix for each word for a particular hearing loss. These new matrices then serve as test data for the previously trained deep neural network, yielding a new classification accuracy. By comparing the reference test accuracy (without any hearing loss) with the new test accuracy, the effect of a particular hearing loss on speech recognition was estimated.

Optionally, Gaussian noise with zero mean and a certain standard deviation, representing somatosen- 
sory input to the DCN, was added independently to the input of each LIF neuron (Figure 1g). Here, the standard deviation corresponds to the noise intensity. As described before, again this finally results in a new binary matrix for each wave file, yet corresponding to a particular hearing loss and, in addition, also to a particular set of frequency channel specific noise intensities. Again, all these new matrices serve as test data for the deep neural network. By comparing the reference test accuracy (without any hearing loss and noise) with the new test accuracy, the effect of particular noise intensities on speech recognition with a certain hearing loss was estimated. A sketch of the complete data flow in case of certain hearing loss and additional noise is depicted in Figure 2.

\section{DCN model neurons show phase coupling below $4 \mathrm{kHz}$}

In order to validate our DCN model, we investigate the spike train output of the 30 LIF neurons for different sine wave inputs (Figure 3). As described in the Methods section, the parameters of the LIF neurons are chosen so that the refractory time $(0.25 \mathrm{~ms})$ of the neurons does not allow for firing rates above $4 \mathrm{kHz}$. Thus, one LIF neuron in our model represents approximately ten biological neurons, having individual refractory times above $1 \mathrm{~ms}$.

We find that for stimulus frequencies above $4 \mathrm{kHz}$ and amplitudes of 0.001 the LIF neurons do not spike at all (Figure 3a). In contrast, for a larger amplitude of 0.002 , a rate coding without phase coupling can be observed (Figure 3b). Furthermore, we find that the LIF neurons are sensitive to amplitude modulations also in the frequency range above $4 \mathrm{kHz}$ (Figure 3c, d). Thus, our DCN neurons are designed so that they allow for phase coupling in the frequency range crucial for speech comprehension, as is known from the human auditory system.

\section{Word processing from cochlea to DCN}

In analogy to the auditory system, the complex auditory stimuli representing spoken words (Figures 4a and 2) are transformed in the cochlea into continuous signals in a number of different frequency channels, in our model 30. However, the cochlea does not perform a simple Fourier transform, but rather splits the signal into multiple band pass filtered signals, thereby preserving the complete phase information (Figures $4 \mathrm{~b}$ and 2). The auditory nerve fibers directly transmit this analogue signal to the DCN [62], which is a special feature of the auditory system [61].

The analogue signals are then further transformed into spike train patterns in the DCN (Figures 2 and $4 \mathrm{c}$ ). Thus, each spoken word is represented as a unique spiking pattern with a dimensionality of $30 \times N$, where 30 corresponds to the number of frequency channels and $N$ is the sampling rate in $\mathrm{Hz}$ times the word length in seconds. Note that we down-sampled these matrices by a factor of five from $44100 \mathrm{~Hz}$ to $8200 \mathrm{~Hz}$ for deep learning (Figure $4 \mathrm{~d}$ ). This does not affect the phase coupling information in the speech relevant frequency range. In order to analyze speech processing in an impaired auditory system, we simulated a hearing loss in the speech relevant frequency range $(400 \mathrm{~Hz}-4 \mathrm{kHz})$ by decreasing the cochlea output amplitudes by a certain factor. The weakened cochlea outputs and the resulting modified DCN spike train outputs are shown in Figure 4b and c, where orange corresponds to an exemplary hearing loss of $30 \mathrm{~dB}$, and blue corresponds to the undisturbed signals, i.e. without hearing loss. The corresponding down sampled spike pattern matrices used as test data for the deep neural network, are shown in Figure 4d (without hearing loss) and in Figure 4e (with $30 \mathrm{~dB}$ hearing loss). We provide an exemplary overview of the effect of different hearing losses from $0 \mathrm{~dB}$ to $45 \mathrm{~dB}$ on the spike pattern matrices in Figure 5. 


\section{Undisturbed test data leads to a reference test accuracy of 0.37}

As described above the complete auditory pathway beyond the DCN, including the superior olive, lateral lemniscus, inferior colliciculus, medial geniculate corpus, and the auditory cortex, is modeled as a deep neural network which is trained on the classification of 207 different German words (custommade data set), or ten English words corresponding to the digits from 0 to 9 (FSDD data set [63]), respectively. In both cases the compressed, i.e. down sampled, DCN output matrices served as training and test data input.

In case of our costum-made data set, the network is exclusively trained on the data of 10 out of 12 speakers, while the remaining two speakers serve as test data. Furthermore, for network training we used only those compressed spike train matrices that correspond to the undisturbed system, i.e. without hearing loss and added noise. Due to the image-like features of the compressed spike pattern matrices, the deep neural network mainly consisted of convolutional layers. The exact architecture is shown in Figure 6a and all parameters are provided in Supplementary Table 1). For training on our costum-made data set, the test accuracy significantly decreases after 20 epochs of training (Figure 6b), and thus we applied the early stopping procedure [64] to prevent the network from overfitting. The trained networks were used for all further analyses with different modifications of the test data set, i.e. different hearing losses and different intensities of intrinsic noise.

\section{Intrinsic noise partially restores spike patterns after simulated hearing loss}

To test the putative beneficial effect of intrinsic noise in case of hearing loss, we analysed spiking patterns generated with and without intrinsic noise and compared them with the corresponding undisturbed patterns (Figure 7). In Figure 7a a sample spike pattern in case of no hearing loss is shown as reference. As expected, a simulated hearing loss of $30 \mathrm{~dB}$ in the frequency range of $400 \mathrm{~Hz}$ to $4 \mathrm{kHz}$ leads to a decreased spiking activity (Figure $7 \mathrm{~b}$ ), which can be partially restored by the addition of intrinsic noise with optimal intensity (Figure 7c).

A point-to-point comparison of the spikes resulting from the undisturbed system (no hearing loss) with the spikes resulting from hearing loss and additional intrinsic noise, demonstrates that there is indeed some improvement. In Figure $7 \mathrm{~d}$ only those spikes are shown that occur in both mentioned cases. In contrast, there are less spikes resulting from hearing loss without intrinsic noise (Figure 7e). Further analysis yield that intrinsic noise not only restores spikes correctly (Figure 7f, yellow), but also introduces false positive spikes (Figure 7f, blue). However, a direct point-to-point comparison of spike patterns does not fully capture the benefit of intrinsic noise. As shown in Figure 7a, c (green boxes), intrinsic noise even restores larger spatio-temporal spiking patterns correctly, yet with some temporal shift.

\section{Intrinsic noise improves accuracy for speech recognition after simulated hearing loss}

We also analyzed the effect of intrinsic noise on speech recognition accuracy in case of hearing loss in different scenarios. Using our costum-made data set, we investigated hearing loss in two different frequency ranges. Furthermore, using the FSDD data set, we investigated hearing loss using two different neural networks. In all cases, we find that intrinsic noise of appropriate intensity improves accuracy for speech recognition after simulated hearing loss.

\section{Costum-made data set and hearing loss in the frequency range of $400 \mathrm{~Hz}$ to $4 \mathrm{kHz}$}

For the first scenario, we used a convolutional neural network (Table 1) trained on our costum-made data set. After training, we simulated a hearing loss in the frequency range of $400 \mathrm{~Hz}$ to $4 \mathrm{kHz}$ 
which is known to be crucial for speech comprehension in humans [65]. The effect of improved or decreased speech comprehension is quantified by the classification accuracy of the words (test accuracy). The classification accuracy as a function of the hearing loss has a biologically plausible sigmoid shape (Figure 8a dark blue curve). The test accuracies as a function of the added noise for different hearing losses show a clear resonance curve with a global maximum (Figure $8 \mathrm{~b}$ ). For a hearing loss of about $20 \mathrm{~dB}$, the relative improvement of speech comprehension is more than doubled (Figure 8c). Furthermore, it can be shown that the optimal noise level correlates with the hearing loss (Figure 8d). This effect is plausible as for a weaker signal a higher noise amplitude is needed to lift the signal over the threshold of the LIF neurons. In summary, it can be stated that the addition of noise can lead to an improved speech comprehension for all hearing losses. This fact can be seen in Figure 8a, where the cyan curve shows the test accuracy as a function of the hearing loss with the ideal amount of added Gaussian noise.

\section{Costum-made data set and hearing loss in the frequency range above $4 \mathrm{kHz}$}

Since many people suffer from hearing losses in the high frequency range [66]. In the next step, the stochastic resonance effect is analyzed for a high frequency range hearing loss starting at a frequency of $4 \mathrm{kHz}$. It can be shown that the high frequency loss does not affect the speech comprehension abilities in the same manner as hearing losses in the critical frequency range between $400 \mathrm{~Hz}$ and $4 \mathrm{kHz}$ (Figure 9a). The relative accuracy does not drop below a value of $50 \%$. Thus, the effect of stochastic resonance is also reduced (Figure 9b), which means a maximal relative improvement of approximately $10 \%$ (Figure 9c, d). Furthermore, there is no real resonance curve with one maximum at a certain noise frequency but a second maximum at a higher noise level (Figure 9b). To put it in nutshell, we can state that the addition of noise can lead to a significant improvement of speech comprehension.

\section{FSDD data set and hearing loss in the frequency range above $400 \mathrm{~Hz}$}

In order to demonstrate that this effect is not limited to a certain data set, language or neural network architecture, we repeated our analyses using two further neural networks, a convolutional neural network (Supplementary Table 2) and a network with Long-Short-Term-Memories (Supplementary Table 3), both trained and tested with the FSDD data set (Figure 10). A hearing loss in the critical frequency range for speech comprehension leads to a decrease in the classification accuracy (10a for the convolutional network and 10c for the Long-Short-Term-Memory network). Furthermore, the stochastic resonance effect in terms of a clear resonance curve with one maximum can be observed (Figure 10b, d). 


\section{Discussion}

In this study, we demonstrated with a computational model of the auditory system that noise added to the DCN may improve speech recognition after hearing loss, by means of SR. The relative benefit of SR turned out to be largest for hearing losses between 20 and $30 \mathrm{~dB}$.

Because SR works by partly restoring missing information in the input data, adding noise improves the classification accuracy of the neural network even after the training period is finished. This stands in contrast to machine learning techniques that achieve an increased robustness and generalization ability by purposefully using noisy training data from the beginning [67], or by adding artificial noise during the training period $[68,69]$.

In our work, we first train the neural network for speech recognition, then simulate a hearing loss, and finally reduce this loss by adding noise. This approach is biologically plausible, as also the brain is trained on speech recognition during childhood [70,71], where hearing ability is usually optimal (Indeed, hearing impairment in childhood can lead to problems in language acquisition, which cannot be fully cured in adulthood [72]). In the coarse of a lifetime, hearing ability becomes permanently $[73,74]$ or temporary worse [75], often due to high amplitude sound exposure.

We have proposed that hearing ability can be restored by a control cycle embedded in the brainstem, along the auditory pathway, which uses internal neural noise to exploit the effect of stochastic resonance [22]. Thus, it is supposed that the neural activity in damaged frequency channels is up-regulated by internally generated noise to restore hearing within this frequency range.

Overshooting of this noise up-regulation is proposed to be the origin of tinnitus [22]. Our model could provide an interesting explanation for overshooting internal noise: In our simulation of high frequency hearing loss, we found that the accuracy as a function of the added noise has not only a single maximum, as expected for a resonance curve, but features a second maximum at a higher noise level (Figure 9b). If the neural control cycle would be drawn to this secondary maximum, this might explain an overshooting of the neural noise and the corresponding emergence of tinnitus [21,22].

Another potential cause of tinnitus arises from the fact that phase locking, the encoding of a signal's phase information in neural spike trains, is only possible for frequencies up to $4 \mathrm{kHz}$, the maximum spike rate of the DCN neurons (Figure 3a).

The stochastic resonance effect probably works only below this limit frequency, and thus it is not clear whether (or how) the neural control system compensates for the hearing loss in the frequency range above $4 \mathrm{kHz}$, as it has no real maximum to optimize for. Potentially, the tuning of the noise parameters in this frequency regime is done only by random trial. This model would fit to the observation that tinnitus mainly occurs in the high frequency range [23].

We were able to show that neural noise could potentially help to increase speech comprehension in neural systems in a computational model of the auditory pathway. An improvement of up to a factor of 2 is possible. This model provides new insights how the auditory system optimizes speech comprehension on small time scales. Furthermore, we could give a mechanistic explanation of the development and characteristics of tinnitus perception. These finding could have a major impact on medical treatment of phantom perceptions, but on the other hand raises new research questions in the field of engineering. It would be interesting to implement these principles to improve artificial systems trained on speech comprehension. Furthermore, the effect of stochastic resonance could be used to improve sensory systems [21].

Our study provides evidence that an interplay of machine learning and neuroscience helps on the one hand to raise understanding of the function of biological neural networks (e.g $[3,76,77])$, an emerging science strand referred to "cognitive computational neuroscience" [78]. On the other hand, basic principles from nature - such as stochastic resonance- can be derived to improve artificial neural systems, which is called "machine behavior" [79]. 


\section{Methods}

\section{Computational Resources}

The simulations were run on a desktop computer equipped with an i9 extreme processor (Intel) with 10 calculation cores. Furthermore, the machine learning was run on the same computer on two Nvidia Titan XP graphical processor units. To test the validity of our calculations the simulations were performed on two different code bases. The main results based on our own speech data set are mainly based on Numpy [80] and SciPy [81] calculations. The convolutional network was implemented in Keras [82] with Tensorflow [83] back-end. All main results were confirmed by analyzing a standard speech data set-the so called Jakobovski free spokeb digit data set (FSDD) [63], containing spoken numbers from 0 to 9 in English language in accordance to the MNIST data set with written digits in this range [84]. This was done using a completely new code base exclusively build of KERAS layers. Thus, a custom-made KERAS layer implemented as sinc FIR filters for the cochlea layer as well as the leaky-integrate-and-fire neurons were implemented. All plots were created using the Matplotlib Python library [85] and plots were arranged using the pylustrator [86].

\section{Cochlea model}

The cochlea is simulated as 30 butterworth bandpass filters (3rd order) with no overlapping bands. These 30 bandpass filters are a simplification of the more than 3000 inner hair cells of the human cochlea [87]. In contrast to other complex cochlea models [88,89], this simplification of the dynamics of the inner hair cells was chosen to derive basic principles and to increase interpretability. The center frequencies (of the bandpass filters) are between $100 \mathrm{~Hz}$ (minfreq.) and $10 \mathrm{kHz}$ (maxfreq.) including the complete frequency range needed for speech comprehension. The center frequencies are chosen to grow exponentially (centerfreq. $=$ minfreq. $\cdot$ factor $^{i}$ with $\mathrm{i} \in\{0,1, \ldots, 29\}$ and factor $=$ maxfreq. $^{1 /(\text { channels }-1)}$ ). Thus, for higher frequencies the spacing of the center frequencies becomes larger in analogy to the tonotopy of the human cochlea $[61,65]$. The width of the bandpass filters is defined as [centerfreq. $\cdot$ factor $^{-0.5}$, centerfreq. factor $\left.^{0.5}\right]$.

\section{Dorsal cochlear nucleus model}

The dorsal cochlear nucleus (DCN) was modeled as 30 leaky integrate-and-fire (LIF) neurons [60], each of these neurons is connected to one frequency channel of the cochlea. Thus, no lateral inhibition was realized to focus on the core effects. The maximum spiking rate of the simulated LIF neurons is approximately $4 \mathrm{kHz}\left(t_{\text {refrac. }}=\frac{11}{44100 \frac{1}{\mathrm{~s}}} \approx \frac{1}{4000} \mathrm{~s}=0.25 \mathrm{~ms}, t_{\text {refrac. }}\right.$ : refractory time $)$, which is much more than the maximum spiking rate of a biological neuron $(250 \mathrm{~Hz})$ [90]. Thus, in the simulation one LIF neuron represent approximately 16 real neurons. The recruitment of several neurons to increase the frequency range in which phase coupling is possible is a core concept within the dorsal cochlear nucleus [91]. The numerical integration of the LIF neurons was performed using the "Euler" method, as this method lead to the lowest computational complexity compared to "Heun" and "Runge Kutte" - being standard integration techniques [92] — without causing significant inaccuracies.

\section{Brain stem and cortex model}

The neural processing stages of the auditory pathway above the DCN including superior olive, lateral lemniscus, inferior colliculus, medial corpus geniculatum in the thalamus, and auditory cortex are modeled as a deep neural network [61]. For our main simulations we used a Deep Convolutional Neural Network [93] to gain independence from translations of the data (for architecture see Fig. 6a, 
for exact parameters cf. Supplements Tab. 1). Furthermore, we used also Deep LSTM networks [94] to double-check the validity and universality of the beneficial effects of intrinsic noise (Supplements Table 3).

\section{Data sets for speech recognition}

\section{Custom-made data set}

Our custom-made data set was recorded from 12 different speakers (6 male, 6 female) in a range of 20-61 years. The data was recorded with a sampling rate of $44.1 \mathrm{kHz}$ bit using Audacity. Each participant had to speak the 207 most common German words 10 times each. After recording the data was labeled using forced alignment and cut into $1 \mathrm{~s}$ intervals. The data from 10 participants served as training data set, whereas the data from the two other speakers was used as test data set. All evaluations, i.e. simulated hearing loss and effect of intrinsic noise, were based on the modified test data.

\section{FSDD data set}

The second used data set is an open data set consisting of spoken digits (0-9) -in analogy to the MNIST data set- in English. The data set is sampled with $8 \mathrm{kHz}$ and consists of 2000 recorded digits from 4 speakers [63]. Here the first five repetitions of for each speaker and each digit are used as test data, the respective remaining 45 repetitions serve as training data. 


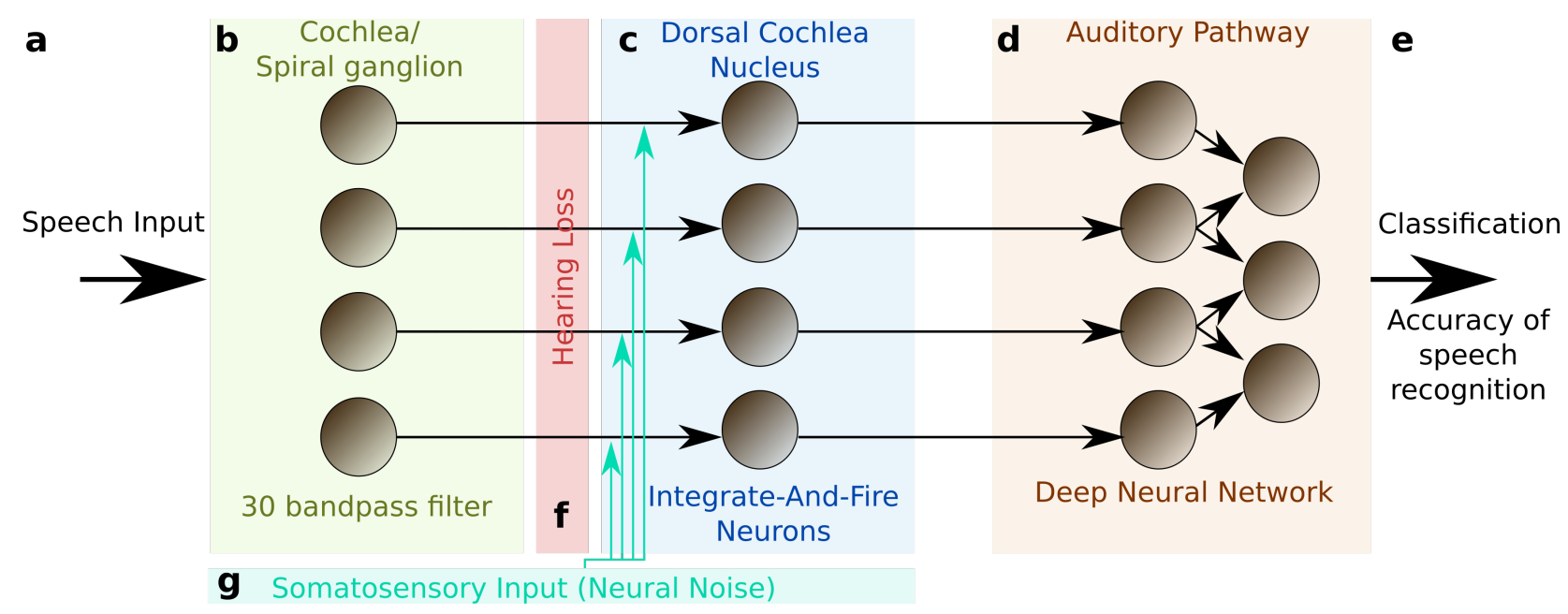

Figure 1: Model layout

The complete model consists of three different modules representing different stages of the auditory pathway in the human brain. The input to the model are single words encoded as wave files with a sampling rate of $44.1 \mathrm{kHz}$ and $1 \mathrm{~s}$ duration (a). The cochlea and the spiral ganglion are modeled as an array of 30 band-pass filters (b). The continuous output signal of (b) serves as input to 30 leaky integrate-and-fire-neurons representing the DCN (c). The spike-train output of the DCN model is down sampled and serves as input for a deep neural network that is trained with error backpropagation on the classification of 207 different german words (d). The classification accuracy serves as a proxy for speech recognition (e). In order to investigate the effect of a particular hearing loss, the cochlea output amplitude is decreased by a certain factor independently for all frequency channels (f). White noise representing somatosensory input to the DCN can be added independently to the input of the different leaky-integrate-and-fire-neurons (LIF, g). 


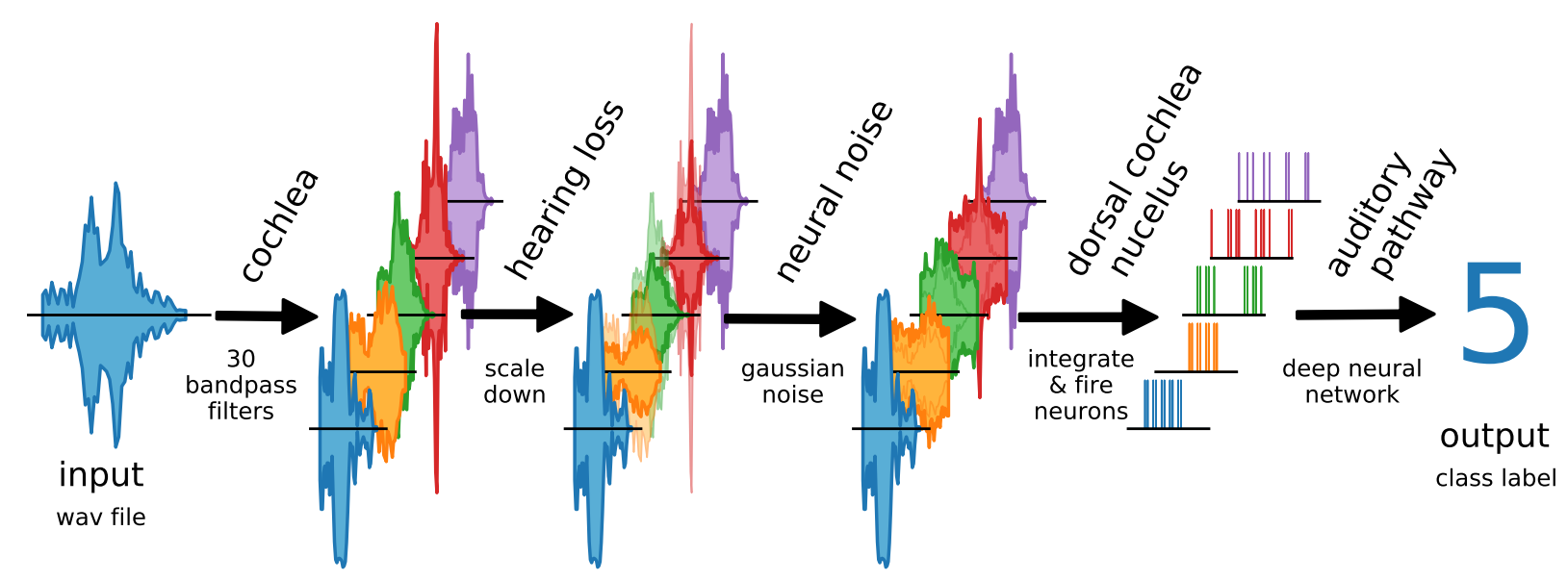

Figure 2: Data flow in auditory pathway model

The scheme shows how the speech data is processed within the model. The cochlea splits the signal via 30 bandpass filters. The bandpass filtered data is scaled down to simulate a hearing loss. The hearing loss affects only channels within the speech relevant frequency range (orange, green, red). The other frequency channels are unchanged. Neural noise is added to investigate the effect of stochastic resonance (only in hearing impaired channels). The DCN is simulated as 30 LIF neurons. Each LIF neuron represents a complete biological neuron population. The spike data is down-sampled and fed to the deep neural network. 

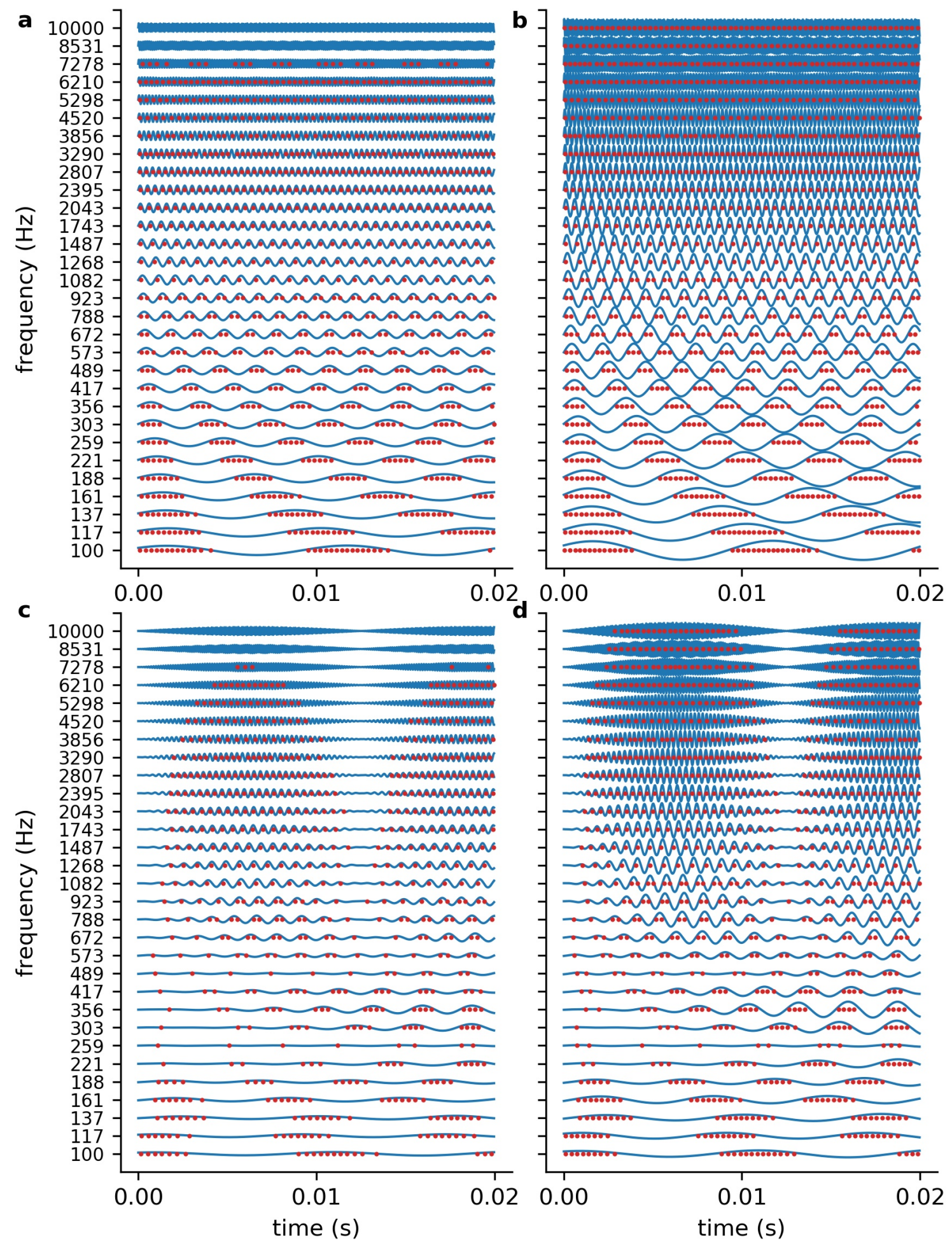

Figure 3: DCN model response to sine waves

Shown are the spiking outputs of the LIF neurons for sine input with two different constant amplitudes (a: 0.001, b: 0.002), and two different amplitude modulations (c, d). For lower amplitudes (a) and higher frequencies the LIF neurons do not spike at all, whereas for higher amplitudes a rate code can be observed as the neurons' maximum spiking rate is limited due to the refractory period. The parameters of the LIF neurons are chosen so that there is phase coupling in the frequency range which is relevant for speech perception. 


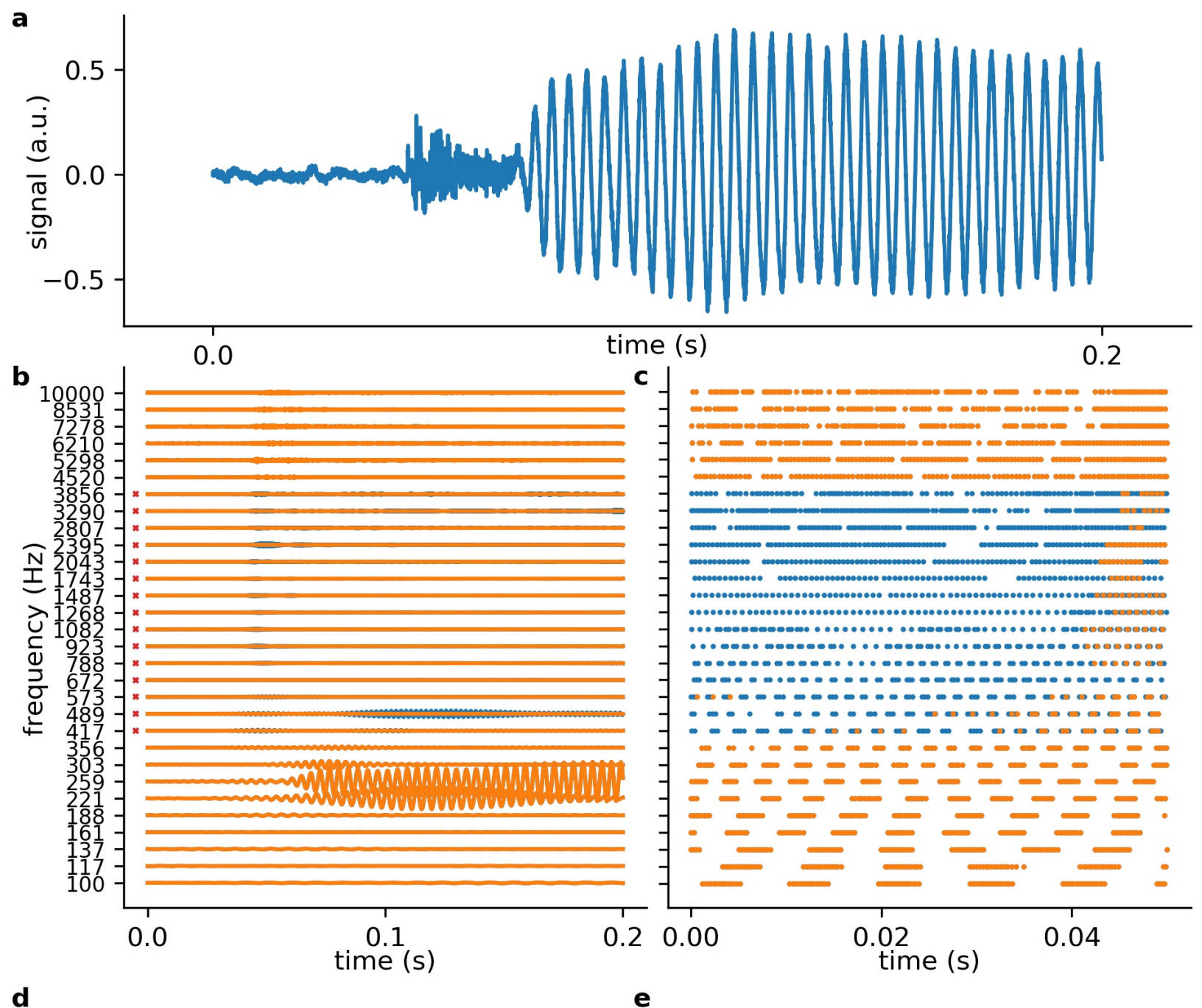

d
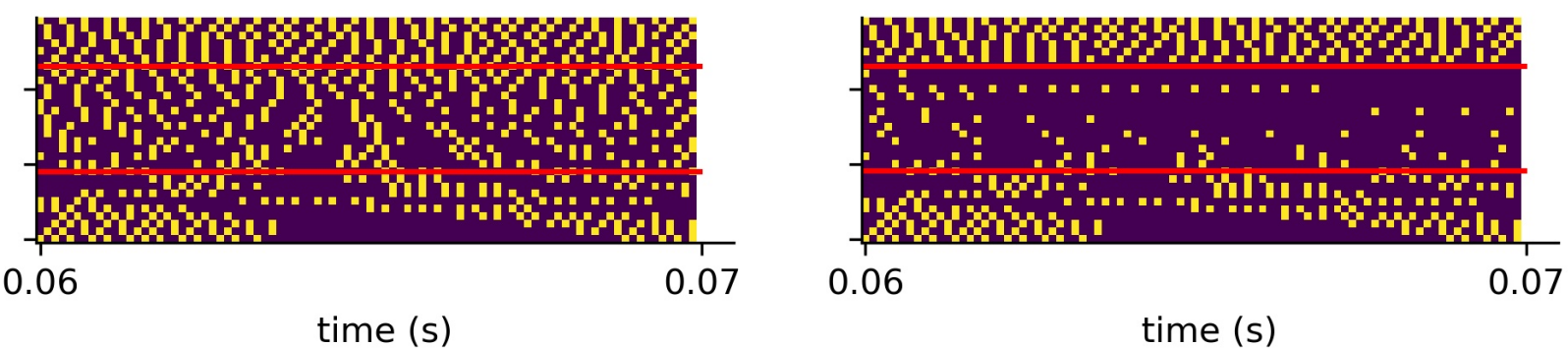

Figure 4: Exemplary processing of a word in cochlea and DCN model

a: The first $0.2 \mathrm{~s}$ of audio data of the German word "die" (the). b) The 30 frequency components (blue without hearing loss, orange with hearing loss) after the first part of the model, which represents the cochlea and the spiral-ganglion (Fig. 1a). A virtual hearing loss is applied by weakening the signal at a certain frequency range (e.g. $400 \mathrm{~Hz}-4 \mathrm{kHz},-30 \mathrm{~dB}$ ). The bandpass filtered signal (matrix of 30 frequency channels and $f_{s} \times$ signal duration) is fed to the LIF neurons (refractory time: $\approx 0.25 \mathrm{~ms}$ ) and spike trains (c) are generated. These spike trains are down-sampled by a factor of 5 and fed to the deep neural network (d). e: The same signal (of d) with added hearing loss of $30 \mathrm{~dB}$ in the frequency range $400 \mathrm{~Hz}-4 \mathrm{kHz}$ being the speech relevant range. 

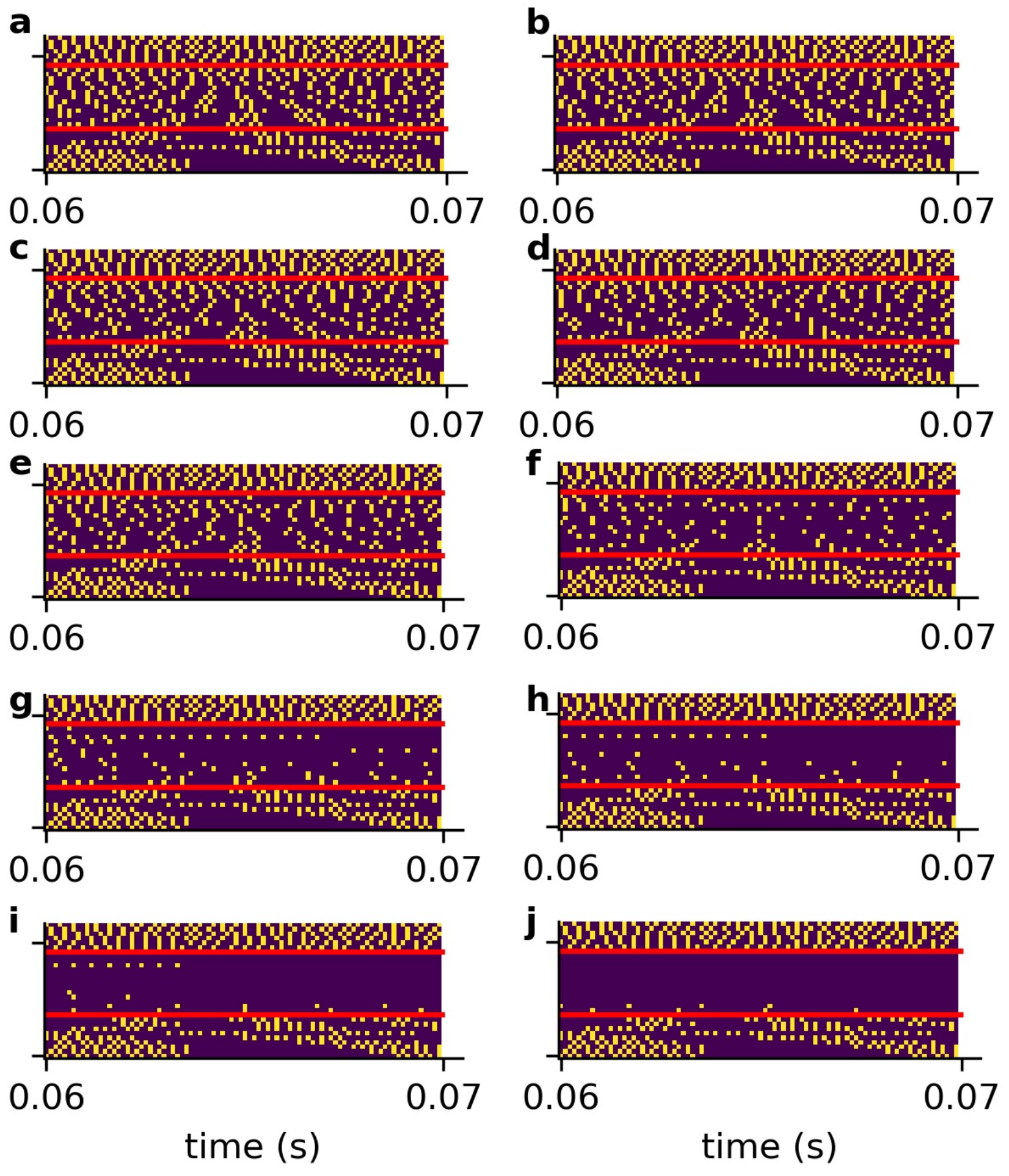

time (s)

Figure 5: Compressed spike patterns with added hearing loss

The figure shows the down-sampled spike patterns of the same word as shown in Figure 4. The speech relevant frequency range $(400 \mathrm{~Hz}-4 \mathrm{kHz})$ is artificially weakened (hearing loss). (a-j) refer to hearing losses $0 \mathrm{~dB}-45 \mathrm{~dB}$. 

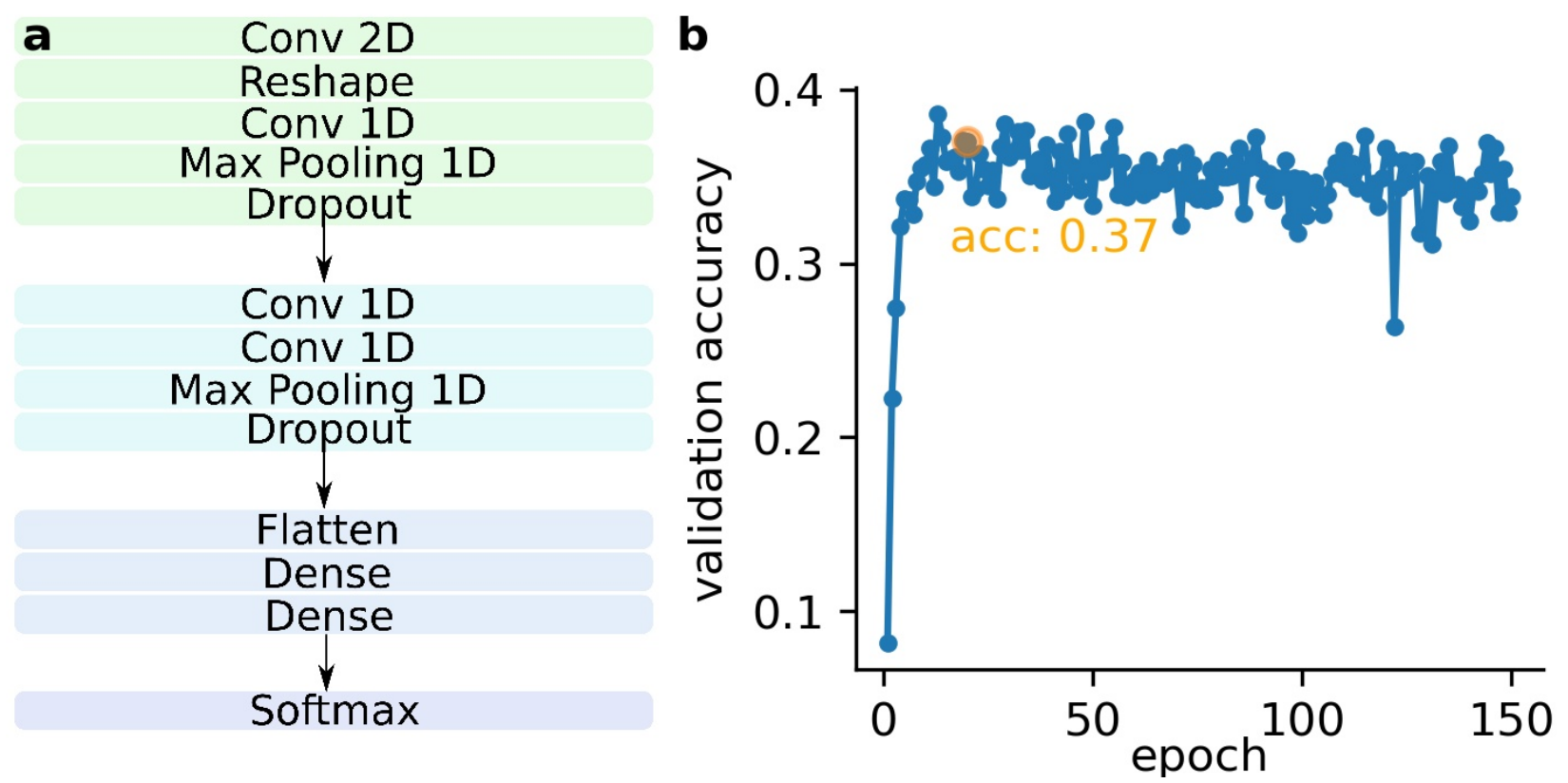

Figure 6: DNN architecture and validation accuracy as function of the epochs

a: Complete network architecture; The network consists of two convolutional network blocks. Each convolutional network block consists of two convolutional layers (ReLu activation), a dropout layer and a maxPooling layer. The convolutional blocks are followed by two fully connected layers (dense layers) with ReLu activation. The last layer is one further fully connected layer with softmax activation (classification layer, for exact architecture see Tab. 1). b: Validation accuracy as function of the epochs; The validation accuracy becomes worse after about 20 epochs (overfitting). The network trained for 20 epochs is used for further analysis. 
a

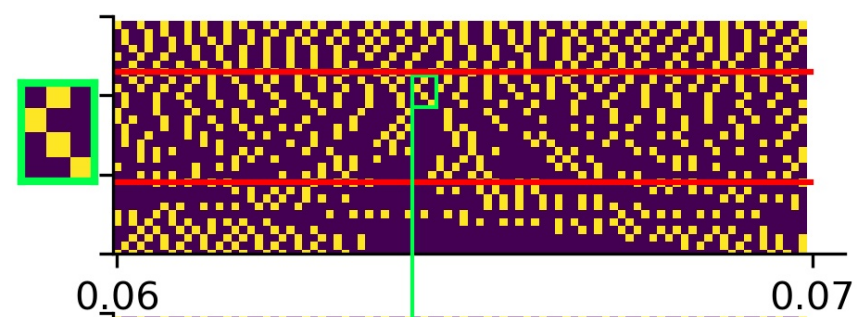

70hны

C
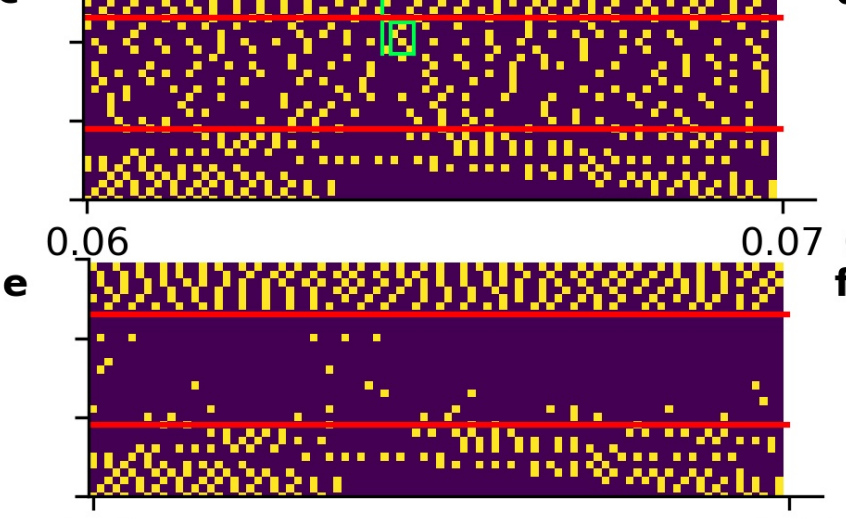

$0.06 \quad$ time $(\mathrm{s})$ b

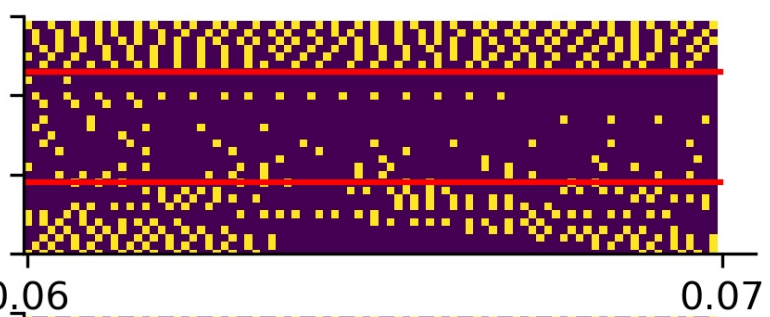

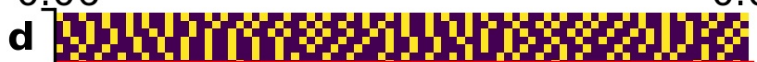
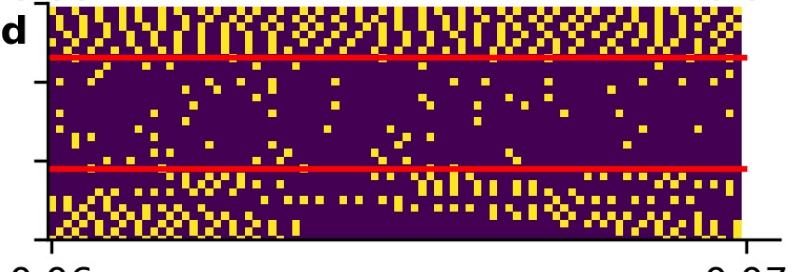

Figure 7: Effect of intrinsic noise on the DCN output patterns.

a: Spiking without HL (same as in Figure 5a). b: Spiking with a HL of $30 \mathrm{~dB}$ (same as Figure $5 \mathrm{~g}$ ). c: Spiking activity with $\mathrm{HL}$ and intrinsic noise of optimal intensity. Additional white noise increases spiking activity. d: Point-to-point comparison of spiking patterns for no $\mathrm{HL}$ and with $\mathrm{HL}$ and intrinsic noise. Shown are only spikes that occur in both cases, i.e. that are not affected by HL or that are correctly restored by noise. e: Point-to-point comparison of spiking patterns for no HL with HL and without intrinsic noise. Shown are only spikes that occur in both cases, i.e. that are not affected by HL. f: Intrinsic noise of optimal intensity not only restores spikes correctly (yellow), but also introduces false positive spikes (dark blue). Intrinsic noise restores spatio-temporal spiking patterns correctly, yet with some temporal shift (green boxes in a and c, zoom of spike pattern in green box). 


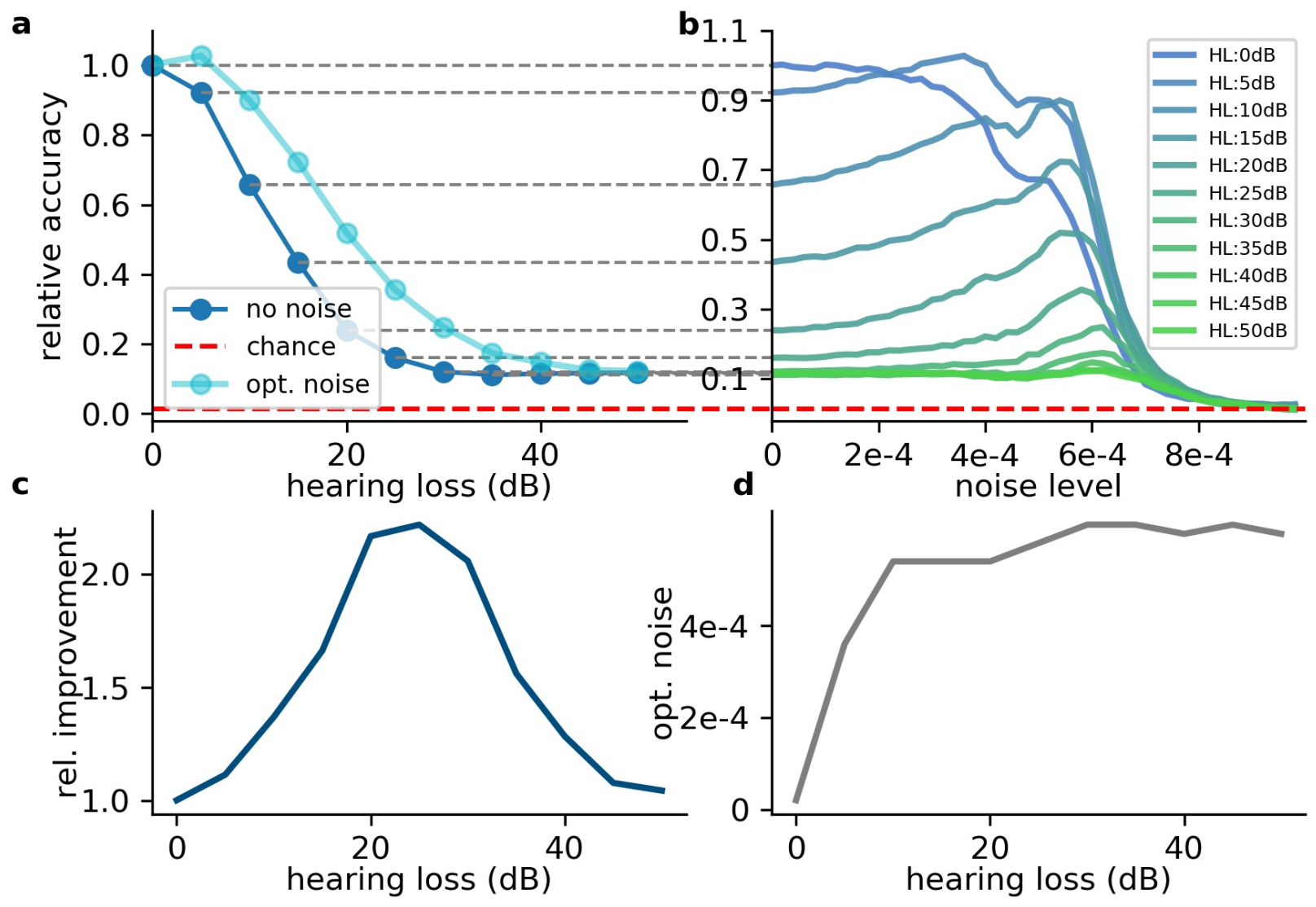

Figure 8: Effect of SR on speech recognition

a: The curve shows the relative accuracy of the trained neural network as a function of the hearing loss (red dashed line: chance level; $\left.\frac{(1 / 207)}{\max \text { accuracy }}\right)$. The hearing loss $(5-50 \mathrm{~dB}, 5 \mathrm{~dB}$ steps, frequency range of HL: $400 \mathrm{~Hz}-4000 \mathrm{~Hz}$ ) was implemented in the test data set and propagated through the pre-trained network. Thus, the cochlea output was multiplied with an attenuation factor $\left(10^{\frac{-\mathrm{HL}}{20}}\right)$. This output was then transformed using the integrate-and-fire neurons and fed in the neural network. b: Relative accuracy as a function of the applied noise level for different hearing losses. Resonance curves with one global maximum at a certain noise level $>0$ could be shown. c: Best relative improvement as a function of the hearing loss. $\mathrm{d}$ : Optimal noise level as a function of the hearing loss 


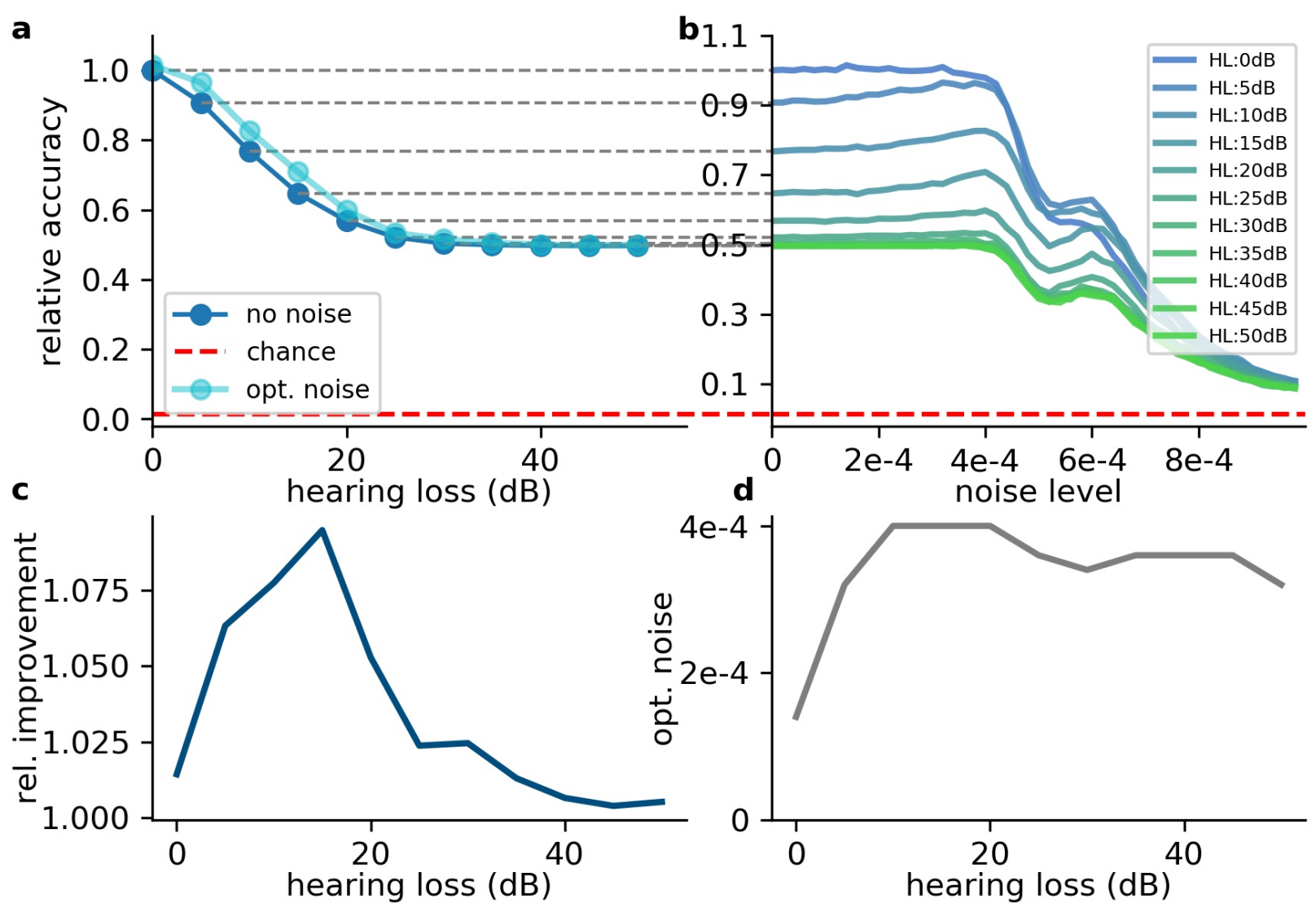

Figure 9: Effect of SR on speech recognition (high frequency hearing loss)

Same analysis as shown in Figure 8 for high frequency hearing loss. a: The plots show the relative accuracy of the trained neural network as a function of the hearing loss (red dashed line: chance level). The high frequency hearing loss lead to different effects $(10-50 \mathrm{~dB}, 10 \mathrm{~dB}$ steps, frequency range of HL: above $4000 \mathrm{~Hz}$ ). b: The relative accuracy as a function of the noise has no clear maximum above the value for no added noise (nearly no SR). Furthermore, a second local maximum occurs. c: The best relative improvement does not significantly increase over $10 \%$. d: Optimal noise level as a function of hearing loss shows similar behavior as for the hearing loss in the speech relevant frequency range (cf. Figure 8). 


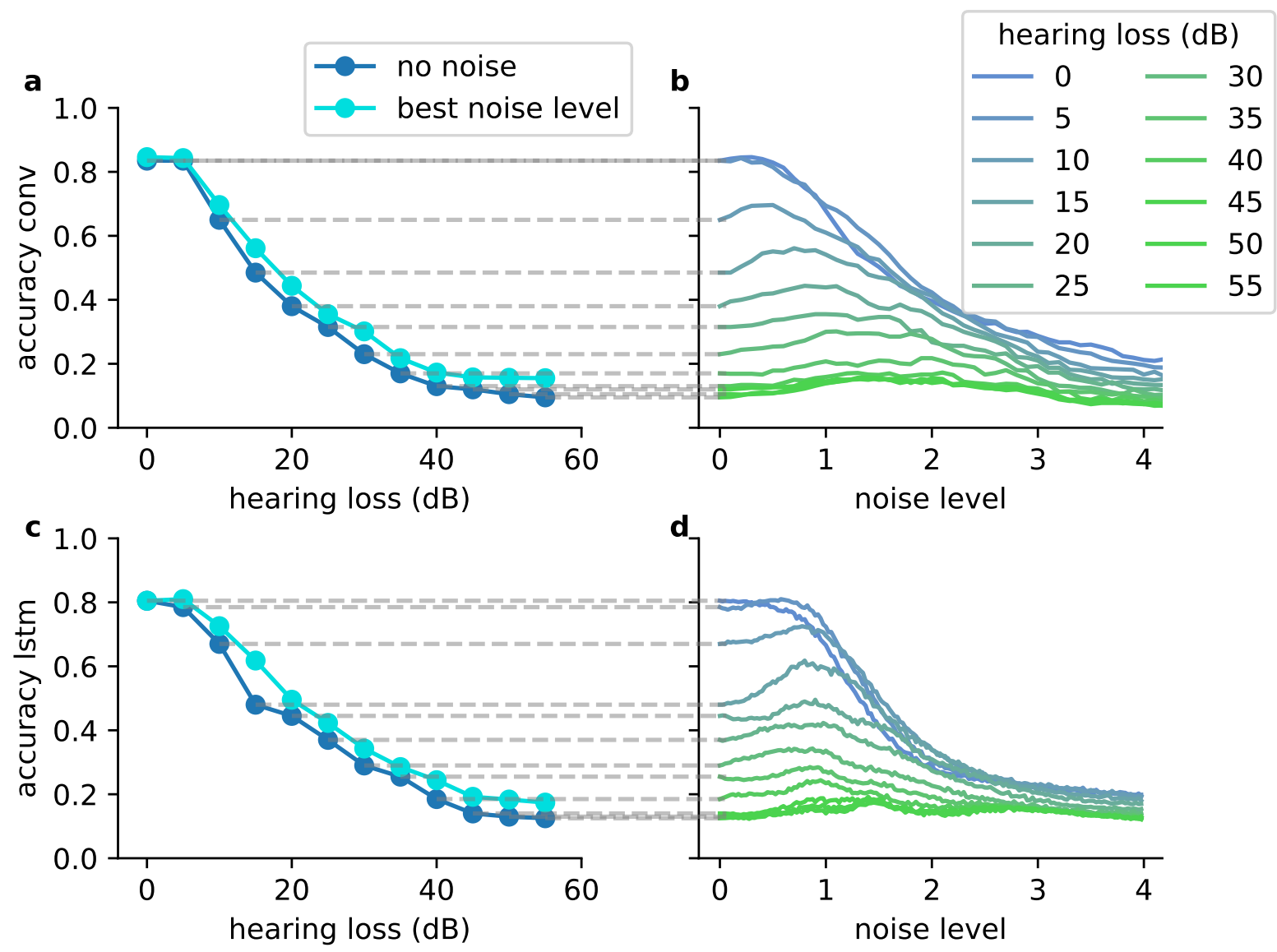

Figure 10: The SR resonance effect in different network architectures using the FSDD data set a: The plot shows the test accuracy as a function of the applied hearing loss for a deep convolutional network architecture (dark blue, starting at $400 \mathrm{~Hz}$, exact network architecture shown in Table 2) trained on English words (digits: 0-9). The impaired speech comprehension by the hearing loss can be partly compensated by adding Gaussian noise (stochastic resonance). The cyan curve shows the improvement of speech comprehension for the optimal noise level (maxima values in $b$ ). b: Test accuracy for different hearing losses (shades of blue) as a function of the added noise. The maxima show that SR can help to restore speech comprehension. c: Similar analysis as shown in (a) for a two layer LSTM network (exact network architecture shown in Table 3); d: Similar analysis as shown in (b) for the LSTM architecture. The improvement of speech perception in impaired systems (hearing loss) is a universal principle and does not depend on the used neural network. 


\section{Additional Information}

\section{Competing interests}

The authors declare no competing financial interests.

\section{Acknowledgments}

This work was funded by the Deutsche Forschungsgemeinschaft (DFG, German Research Foundation): grant KR5148/2-1 to PK - project number 436456810, and the Emergent Talents Initiative (ETI) of the University Erlangen-Nuremberg (grant 2019/2-Phil-01 to PK), and the Interdisciplinary Center for Clinical Research (IZKF) at the University Hospital of the University ErlangenNuremberg (grant ELAN-17-12-27-1-Schilling to AS).

The authors are grateful for the donation of two Titan Xp GPUs by the NVIDIA Corporation.

We thank Martin Haller for technical assistance, and Holger Schulze for providing us access to the lab.

Finally, we wish to thank the twelve speakers who lend us their voices for generating the costummade speech data set.

\section{Author contributions}

PK and AS designed the study. AZ created the costum-made data set. AS, RG and PK implemented the model. AS, RG, CM, AM and PK discussed the results. AS, PK, RG, CM and AM wrote the manuscript. 


\section{References}

[1] Mark D McDonnell and Derek Abbott. What is stochastic resonance? definitions, misconceptions, debates, and its relevance to biology. PLoS computational biology, 5(5):e1000348, 2009 .

[2] Arkady S Pikovsky and Jürgen Kurths. Coherence resonance in a noise-driven excitable system. Physical Review Letters, 78(5):775, 1997.

[3] Patrick Krauss, Karin Prebeck, Achim Schilling, and Claus Metzner. Recurrence resonance" in three-neuron motifs. Frontiers in computational neuroscience, 13, 2019.

[4] Roberto Benzi, Alfonso Sutera, and Angelo Vulpiani. The mechanism of stochastic resonance. Journal of Physics A: mathematical and general, 14(11):L453, 1981.

[5] Luca Gammaitoni, Peter Hänggi, Peter Jung, and Fabio Marchesoni. Stochastic resonance. Reviews of modern physics, 70(1):223, 1998.

[6] Frank Moss, Lawrence M Ward, and Walter G Sannita. Stochastic resonance and sensory information processing: a tutorial and review of application. Clinical neurophysiology, 115(2):267$281,2004$.

[7] Kurt Wiesenfeld and Frank Moss. Stochastic resonance and the benefits of noise: from ice ages to crayfish and squids. Nature, 373(6509):33, 1995.

[8] John K Douglass, Lon Wilkens, Eleni Pantazelou, and Frank Moss. Noise enhancement of information transfer in crayfish mechanoreceptors by stochastic resonance. Nature, 365(6444):337, 1993.

[9] JAMES J Collins, THOMAS T Imhoff, and PETER Grigg. Noise-enhanced information transmission in rat sa1 cutaneous mechanoreceptors via aperiodic stochastic resonance. Journal of Neurophysiology, 76(1):642-645, 1996.

[10] Bruce J Gluckman, Theoden I Netoff, Emily J Neel, William L Ditto, Mark L Spano, and Steven J Schiff. Stochastic resonance in a neuronal network from mammalian brain. Physical Review Letters, 77(19):4098, 1996.

[11] Daichi Nozaki, Douglas J Mar, Peter Grigg, and James J Collins. Effects of colored noise on stochastic resonance in sensory neurons. Physical Review Letters, 82(11):2402, 1999.

[12] Marius Usher and Mario Feingold. Stochastic resonance in the speed of memory retrieval. Biological Cybernetics, 83(6):L011-L016, 2000.

[13] Lawrence M Ward, Alexander Neiman, and Frank Moss. Stochastic resonance in psychophysics and in animal behavior. Biological cybernetics, 87(2):91-101, 2002.

[14] Bart Kosko and Sanya Mitaim. Stochastic resonance in noisy threshold neurons. Neural networks, 16(5-6):755-761, 2003.

[15] Takatsugu Aihara, Keiichi Kitajo, Daichi Nozaki, and Yoshiharu Yamamoto. Internal noise determines external stochastic resonance in visual perception. Vision research, 48(14):1569$1573,2008$. 
[16] A Aldo Faisal, Luc PJ Selen, and Daniel M Wolpert. Noise in the nervous system. Nature reviews neuroscience, 9(4):292, 2008.

[17] Hiroyuki Mino. The effects of spontaneous random activity on information transmission in an auditory brain stem neuron model. Entropy, 16(12):6654-6666, 2014.

[18] Sanya Mitaim and Bart Kosko. Adaptive stochastic resonance. Proceedings of the IEEE, 86(11):2152-2183, 1998.

[19] Gregor Wenning and Klaus Obermayer. Activity driven adaptive stochastic resonance. Physical review letters, 90(12):120602, 2003.

[20] Sanya Mitaim and Bart Kosko. Adaptive stochastic resonance in noisy neurons based on mutual information. IEEE Transactions on Neural Networks, 15(6):1526-1540, 2004.

[21] Patrick Krauss, Claus Metzner, Achim Schilling, Christian Schütz, Konstantin Tziridis, Ben Fabry, and Holger Schulze. Adaptive stochastic resonance for unknown and variable input signals. Scientific reports, 7(1):2450, 2017.

[22] Patrick Krauss, Konstantin Tziridis, Claus Metzner, Achim Schilling, Ulrich Hoppe, and Holger Schulze. Stochastic resonance controlled upregulation of internal noise after hearing loss as a putative cause of tinnitus-related neuronal hyperactivity. Frontiers in neuroscience, 10:597, 2016.

[23] Dominik Gollnast, Konstantin Tziridis, Patrick Krauss, Achim Schilling, Ulrich Hoppe, and Holger Schulze. Analysis of audiometric differences of patients with and without tinnitus in a large clinical database. Frontiers in Neurology, 8:31, 2017.

[24] Patrick Krauss, Konstantin Tziridis, Achim Schilling, and Holger Schulze. Cross-modal stochastic resonance as a universal principle to enhance sensory processing. Frontiers in neuroscience, 12:578, 2018.

[25] P Krauss, A Schilling, K Tziridis, and H Schulze. Models of tinnitus development: From cochlea to cortex. HNO, 2019.

[26] J Wang, NL Powers, P Hofstetter, P Trautwein, D Ding, and R Salvi. Effects of selective inner hair cell loss on auditory nerve fiber threshold, tuning and spontaneous and driven discharge rate. Hearing research, 107(1-2):67-82, 1997.

[27] Sönke Ahlf, Konstantin Tziridis, Sabine Korn, Ilona Strohmeyer, and Holger Schulze. Predisposition for and prevention of subjective tinnitus development. PLoS One, 7(10):e44519, 2012 .

[28] Konstantin Tziridis, Sönke Ahlf, Marcus Jeschke, Max FK Happel, Frank W Ohl, and Holger Schulze. Noise trauma induced neural plasticity throughout the auditory system of mongolian gerbils: differences between tinnitus developing and non-developing animals. Frontiers in neurology, 6:22, 2015.

[29] Calvin Wu, Roxana A Stefanescu, David T Martel, and Susan E Shore. Tinnitus: maladaptive auditory-somatosensory plasticity. Hearing research, 334:20-29, 2016.

[30] Andrew J Heller. Classification and epidemiology of tinnitus. Otolaryngologic Clinics of North America, 36(2):239-248, 2003. 
[31] Ovidiu König, Roland Schaette, Richard Kempter, and Manfred Gross. Course of hearing loss and occurrence of tinnitus. Hearing research, 221(1-2):59-64, 2006.

[32] Jeffrey J Nelson and Kejian Chen. The relationship of tinnitus, hyperacusis, and hearing loss. Ear, nose $\&$ throat journal, 83(7):472-476, 2004.

[33] Susan E Shore, Larry E Roberts, and Berthold Langguth. Maladaptive plasticity in tinnitus - triggers, mechanisms and treatment. Nature Reviews Neurology, 12(3):150, 2016.

[34] Roland Schaette and David McAlpine. Tinnitus with a normal audiogram: physiological evidence for hidden hearing loss and computational model. Journal of Neuroscience, 31(38):1345213457, 2011.

[35] Leslie D Liberman and M Charles Liberman. Dynamics of cochlear synaptopathy after acoustic overexposure. Journal of the Association for Research in Otolaryngology, 16(2):205-219, 2015.

[36] James A Kaltenbach, Donald A Godfrey, John B Neumann, Devin L McCaslin, Chad E Afman, and Jinsheng Zhang. Changes in spontaneous neural activity in the dorsal cochlear nucleus following exposure to intense sound: relation to threshold shift. Hearing research, 124(1-2):7884, 1998.

[37] James A Kaltenbach and Chad E Afman. Hyperactivity in the dorsal cochlear nucleus after intense sound exposure and its resemblance to tone-evoked activity: a physiological model for tinnitus. Hearing research, 140(1-2):165-172, 2000.

[38] Mark A Zacharek, James A Kaltenbach, T Alecia Mathog, and Jinsheng Zhang. Effects of cochlear ablation on noise induced hyperactivity in the hamster dorsal cochlear nucleus: implications for the origin of noise induced tinnitus. Hearing research, 172(1-2):137-144, 2002.

[39] TJ Brozoski, CA Bauer, and DM Caspary. Elevated fusiform cell activity in the dorsal cochlear nucleus of chinchillas with psychophysical evidence of tinnitus. Journal of Neuroscience, 22(6):2383-2390, 2002.

[40] James A Kaltenbach, Mark A Zacharek, Jinsheng Zhang, and Sarah Frederick. Activity in the dorsal cochlear nucleus of hamsters previously tested for tinnitus following intense tone exposure. Neuroscience letters, 355(1-2):121-125, 2004.

[41] James A Kaltenbach, John D Rachel, T Alecia Mathog, Jinsheng Zhang, Pamela R Falzarano, and Matthew Lewandowski. Cisplatin-induced hyperactivity in the dorsal cochlear nucleus and its relation to outer hair cell loss: relevance to tinnitus. Journal of neurophysiology, 88(2):699$714,2002$.

[42] Y Gao, N Manzoor, and JA Kaltenbach. Evidence of activity-dependent plasticity in the dorsal cochlear nucleus, in vivo, induced by brief sound exposure. Hearing research, 341:31-42, 2016.

[43] David K Ryugo, Charles-André Haenggeli, and John R Doucet. Multimodal inputs to the granule cell domain of the cochlear nucleus. Experimental brain research, 153(4):477-485, 2003.

[44] Susan E Shore and Jianxun Zhou. Somatosensory influence on the cochlear nucleus and beyond. Hearing Research, 216:90-99, 2006. 
[45] Susanne Dehmel, Shashwati Pradhan, Seth Koehler, Sanford Bledsoe, and Susan Shore. Noise overexposure alters long-term somatosensory-auditory processing in the dorsal cochlear nucleus - possible basis for tinnitus-related hyperactivity? Journal of Neuroscience, 32(5):16601671, 2012.

[46] Chunhua Zeng, Ziheng Yang, Lauren Shreve, Sanford Bledsoe, and Susan Shore. Somatosensory projections to cochlear nucleus are upregulated after unilateral deafness. Journal of Neuroscience, 32(45):15791-15801, 2012.

[47] Rebecca J Pinchoff, Robert F Burkard, Richard J Salvi, Mary Lou Coad, and Alan H Lockwood. Modulation of tinnitus by voluntary jaw movements. The American journal of otology, 19(6):785-789, 1998.

[48] Robert Aaron Levine. Somatic (craniocervical) tinnitus and the dorsal cochlear nucleus hypothesis. American journal of otolaryngology, 20(6):351-362, 1999.

[49] Zheng-Quan Tang and Laurence O Trussell. Serotonergic regulation of excitability of principal cells of the dorsal cochlear nucleus. Journal of Neuroscience, 35(11):4540-4551, 2015.

[50] Zheng-Quan Tang and Laurence O Trussell. Serotonergic modulation of sensory representation in a central multisensory circuit is pathway specific. Cell reports, 20(8):1844-1854, 2017.

[51] SE Shore, S Koehler, M Oldakowski, LF Hughes, and S Syed. Dorsal cochlear nucleus responses to somatosensory stimulation are enhanced after noise-induced hearing loss. European Journal of Neuroscience, 27(1):155-168, 2008.

[52] SE Shore. Plasticity of somatosensory inputs to the cochlear nucleus-implications for tinnitus. Hearing research, 281(1-2):38-46, 2011.

[53] Juan Huang, Thomas Lu, Benjamin Sheffield, and Fan-Gang Zeng. Electro-tactile stimulation enhances cochlear-implant melody recognition: Effects of rhythm and musical training. Ear and Hearing, 2019.

[54] Juan Huang, Benjamin Sheffield, Payton Lin, and Fan-Gang Zeng. Electro-tactile stimulation enhances cochlear implant speech recognition in noise. Scientific reports, 7(1):2196, 2017.

[55] Brian CJ Moore and Brian R Glasberg. Suggested formulae for calculating auditory-filter bandwidths and excitation patterns. The journal of the acoustical society of America, 74(3):750$753,1983$.

[56] Dorian S Houser, David A Helweg, and Patrick WB Moore. A bandpass filter-bank model of auditory sensitivity in the humpback whale. Aquatic Mammals, 27(2):82-91, 2001.

[57] Mark Sayles and Ian M Winter. Equivalent-rectangular bandwidth of single units in the anaesthetized guinea-pig ventral cochlear nucleus. Hearing research, 262(1-2):26-33, 2010.

[58] Joseph B Nadol Jr. Comparative anatomy of the cochlea and auditory nerve in mammals. Hearing research, 34(3):253-266, 1988.

[59] IJ Russell and KE Nilsen. The location of the cochlear amplifier: spatial representation of a single tone on the guinea pig basilar membrane. Proceedings of the National Academy of Sciences, 94(6):2660-2664, 1997. 
[60] Anthony N Burkitt. A review of the integrate-and-fire neuron model: I. homogeneous synaptic input. Biological cybernetics, 95(1):1-19, 2006.

[61] Eric R Kandel, James H Schwartz, Thomas M Jessell, Department of Biochemistry, Molecular Biophysics Thomas Jessell, Steven Siegelbaum, and AJ Hudspeth. Principles of neural science, volume 4. McGraw-hill New York, 2000.

[62] Eric D Young and Kevin A Davis. Circuitry and function of the dorsal cochlear nucleus. In Integrative functions in the mammalian auditory pathway, pages 160-206. Springer, 2002.

[63] Zohar Jackson, César Souza, Jason Flaks, Yuxin Pan, Hereman Nicolas, and Adhish Thite. Jakobovski/free-spoken-digit-dataset: v1.0.8, doi: 10.5281/zenodo.1342401, August 2018.

[64] Rich Caruana, Steve Lawrence, and C Lee Giles. Overfitting in neural nets: Backpropagation, conjugate gradient, and early stopping. In Advances in neural information processing systems, pages 402-408, 2001.

[65] Stuart Ira Fox. Human Physiology 9th Editon. McGraw-Hill press, New York, USA, 2006.

[66] A Ciorba, A Benatti, C Bianchini, Claudia Aimoni, Stefano Volpato, R Bovo, and A Martini. High frequency hearing loss in the elderly: effect of age and noise exposure in an italian group. The Journal of Laryngology \& Otology, 125(8):776-780, 2011.

[67] Vladimir Karpukhin, Omer Levy, Jacob Eisenstein, and Marjan Ghazvininejad. Training on synthetic noise improves robustness to natural noise in machine translation. arXiv preprint arXiv:1902.01509, 2019.

[68] Guozhong An. The effects of adding noise during backpropagation training on a generalization performance. Neural computation, 8(3):643-674, 1996.

[69] Chenyang Zhao, Olivier Siguad, Freek Stulp, and Timothy M Hospedales. Investigating generalisation in continuous deep reinforcement learning. arXiv preprint arXiv:1902.07015, 2019.

[70] Ewa Dabrowska and Wojciech Kubinski. Language acquisition in the light of cognitive linguistics. Žmogus Kalbos Erdveje [Man in the Space of Language]. Moksliniu straipsniu rinkinys, $3(1): 253-267,2004$.

[71] Judit Gervain. Plasticity in early language acquisition: the effects of prenatal and early childhood experience. Current opinion in neurobiology, 35:13-20, 2015.

[72] Hannah Pimperton and Colin R Kennedy. The impact of early identification of permanent childhood hearing impairment on speech and language outcomes. Archives of disease in childhood, $97(7): 648-653,2012$.

[73] Qi Huang and Jianguo Tang. Age-related hearing loss or presbycusis. European Archives of Oto-rhino-laryngology, 267(8):1179-1191, 2010.

[74] George A Gates and John H Mills. Presbycusis. The Lancet, 366(9491):1111-1120, 2005.

[75] James F Willott and Shao-Ming Lu. Noise-induced hearing loss can alter neural coding and increase excitability in the central nervous system. Science, 216(4552):1331-1334, 1982.

[76] Richard C Gerum, André Erpenbeck, Patrick Krauss, and Achim Schilling. Sparsity through evolutionary pruning prevents neuronal networks from overfitting. arXiv preprint arXiv:1911.10988, 2019. 
[77] Achim Schilling, Claus Metzner, Jonas Rietsch, Richard Gerum, Holger Schulze, and Patrick Krauss. How deep is deep enough?-quantifying class separability in the hidden layers of deep neural networks. arXiv preprint arXiv:1811.01753, 2018.

[78] Nikolaus Kriegeskorte and Pamela K Douglas. Cognitive computational neuroscience. Nature neuroscience, 21(9):1148-1160, 2018.

[79] Iyad Rahwan, Manuel Cebrian, Nick Obradovich, Josh Bongard, Jean-François Bonnefon, Cynthia Breazeal, Jacob W Crandall, Nicholas A Christakis, Iain D Couzin, Matthew O Jackson, et al. Machine behaviour. Nature, 568(7753):477-486, 2019.

[80] Stéfan van der Walt, S Chris Colbert, and Gael Varoquaux. The numpy array: a structure for efficient numerical computation. Computing in Science $\&$ Engineering, 13(2):22-30, 2011.

[81] Eric Jones, Travis Oliphant, and Pearu Peterson. Scipy: Open source scientific tools for python. 2001.

[82] Francois Chollet. Deep Learning mit Python und Keras: Das Praxis-Handbuch vom Entwickler der Keras-Bibliothek. MITP-Verlags GmbH \& Co. KG, 2018.

[83] Martín Abadi, Paul Barham, Jianmin Chen, Zhifeng Chen, Andy Davis, Jeffrey Dean, Matthieu Devin, Sanjay Ghemawat, Geoffrey Irving, Michael Isard, et al. Tensorflow: A system for largescale machine learning. In 12th \{USENIX\} Symposium on Operating Systems Design and Implementation (\{OSDI\} 16), pages 265-283, 2016.

[84] Yann LeCun, Léon Bottou, Yoshua Bengio, and Patrick Haffner. Gradient-based learning applied to document recognition. Proceedings of the IEEE, 86(11):2278-2324, 1998.

[85] John D Hunter. Matplotlib: A 2d graphics environment. Computing in science 85 engineering, 9(3):90-95, 2007.

[86] Richard Gerum. pylustrator: code generation for reproducible figures for publication. arXiv preprint arXiv:1910.00279, 2019.

[87] Peter Dallos. The active cochlea. Journal of Neuroscience, 12(12):4575-4585, 1992.

[88] Qing Tan and Laurel H Carney. A phenomenological model for the responses of auditory-nerve fibers. ii. nonlinear tuning with a frequency glide. The Journal of the Acoustical Society of America, 114(4):2007-2020, 2003.

[89] Jordan Chambers, Diego Elgueda, Jonathan B Fritz, Shihab A Shamma, Anthony N Burkitt, and David B Grayden. Computational neural modelling of auditory cortical receptive fields. Frontiers in computational neuroscience, 13:28, 2019.

[90] Lance Nizami. Estimating auditory neuronal dynamic range using a fitted function. Hearing research, 167(1-2):13-27, 2002.

[91] Gerald Langner. Physiological properties of units in the cochlear nucleus are adequate for a model of periodicity analysis in the auditory midbrain. In Auditory Pathway, pages 207-212. Springer, 1988.

[92] Muhammad Faris Fathoni and Aciek Ida Wuryandari. Comparison between euler, heun, rungekutta and adams-bashforth-moulton integration methods in the particle dynamic simulation. In 2015 4th International Conference on Interactive Digital Media (ICIDM), pages 1-7. IEEE, 2015. 
[93] Yann LeCun, Yoshua Bengio, and Geoffrey Hinton. Deep learning. nature, 521(7553):436-444, 2015.

[94] Sepp Hochreiter and Jürgen Schmidhuber. Long short-term memory. Neural computation, 9(8):1735-1780, 1997. 


\section{Supplements}

\begin{tabular}{c||cccc} 
Layer & Type & input-output-dim & activation & characteristics \\
\hline \hline 1 & Convolution 2D & $30,8820,1 ; 1,8791,128$ & ReLu & \\
2 & Reshape & $1,8791,128 ; 8791,128$ & & \\
3 & Convolution 1D & 8791,$128 ; 8782,128$ & ReLu & \\
4 & MaxPooling 1D & 8782,$128 ; 4391,128$ & & poolsize: 2 \\
5 & DropOut & 4391,$128 ; 4391,128$ & & dropout: 0.5 \\
6 & Convolution 1D & 4391,$128 ; 4391,128$ & ReLu & \\
7 & Convolution 1D & 4391,$128 ; 4390,128$ & ReLu & \\
8 & MaxPooling 1D & 4390,$128 ; 2195,128$ & & poolsize: 2 \\
9 & DropOut & 2195,$128 ; 2195,128$ & & dropout: 0.5 \\
10 & Flatten & 2195,$128 ; 280960$ & & \\
11 & Dense & $280960 ; 150$ & ReLu & \\
12 & Dense & $150 ; 50$ & ReLu & \\
13 & Dense & $50 ; 207$ & Softmax &
\end{tabular}

Table 1: Exact paramters of the used deep convolutional network (main analysis)

\begin{tabular}{c||cccc} 
Layer & Type & input-output-dim & activation & characteristics \\
\hline \hline 1 & Convolution 2D & $9131,30,1 ; 9102,1,32$ & relu & \\
2 & MaxPooling 2D & $9102,1,32 ; 4551,1,32$ & & poolsize: $(2,1)$ \\
3 & DropOut & $4551,1,32 ; 4551,1,32$ & & droupout: 0.2 \\
4 & Convolution 2D & $4551,1,32 ; 4547,1,64$ & relu & \\
5 & MaxPooling 2D & $4547,1,64 ; 2273,1,64$ & & poolsize: $(2,1)$ \\
6 & DropOut & $2273,1,64 ; 2273,1,64$ & & droupout: 0.2 \\
7 & Convolution 2D & $2273,1,64 ; 2272,1,32$ & relu & \\
8 & MaxPooling 2D & $2272,1,32 ; 1136,1,32$ & & poolsize: $(2,1)$ \\
9 & DropOut & $1136,1,32 ; 1136,1,32$ & & droupout: 0.2 \\
10 & Flatten & $1136,1,32 ; 36352$ & & \\
11 & Dense & $36352 ; 400$ & relu & \\
12 & DropOut & $400 ; 400$ & & droupout: 0.2 \\
13 & Dense & $400 ; 50$ & relu & \\
14 & Dense & $50 ; 10$ & softmax &
\end{tabular}

Table 2: Exact paramters of the used deep convolutional network (FSDD data set) 


\begin{tabular}{c||cccc} 
Layer & Type & input-output-dim & activation & characteristics \\
\hline \hline 1 & GroupToBatches & 9000,$30 ; 45,6000$ & & \\
2 & LSTM & 45,$6000 ; 45,200$ & tanh & \\
3 & DropOut & 45,$200 ; 45,200$ & & droupout: 0.5 \\
4 & LSTM & 45,$200 ; 45,100$ & tanh & \\
5 & DropOut & 45,$100 ; 45,100$ & & droupout: 0.5 \\
6 & TimeDistributed Dense & 45,$100 ; 45,100$ & & \\
7 & DropOut & 45,$100 ; 45,100$ & & droupout: 0.5 \\
8 & TimeDistributed Dense & 45,$100 ; 45,10$ & &
\end{tabular}

Table 3: Exact paramters of the used LSTM network (FSDD data set) 